\title{
Gilles Bourquin, Théologie de la spiritualité. Une approche protestante de la culture religieuse en postmodernité
}

Genève, Labor et Fides, coll. « Lieux théologiques », 2011, 430 p.

\section{Daniel Vidal}

\section{(2) OpenEdition \\ Journals}

\section{Édition électronique}

URL : http://journals.openedition.org/assr/23454

DOI : $10.4000 /$ assr.23454

ISSN : $1777-5825$

\section{Éditeur}

Éditions de l'EHESS

Édition imprimée

Date de publication : 31 décembre 2011

Pagination : 130

ISBN : 9782713223273

ISSN : 0335-5985

\section{Référence électronique}

Daniel Vidal, « Gilles Bourquin, Théologie de la spiritualité. Une approche protestante de la culture religieuse en postmodernité ", Archives de sciences sociales des religions [En ligne], 156 | octobredécembre 2011, document 156-26, mis en ligne le 14 février 2012, consulté le 21 septembre 2020. URL : http://journals.openedition.org/assr/23454; DOI : https://doi.org/10.4000/assr.23454 


\title{
Gilles Bourquin, Théologie de la spiritualité. Une approche protestante de la culture religieuse en postmodernité
}

Genève, Labor et Fides, coll. « Lieux théologiques », 2011, 430 p.

\author{
Daniel Vidal
}

\section{RÉFÉRENCE}

Gilles Bourquin, Théologie de la spiritualité. Une approche protestante de la culture religieuse en postmodernité, Genève, Labor et Fides, coll. « Lieux théologiques », 2011, $430 \mathrm{p}$.

1 Un cri d'alarme pourra-t-il jamais arrêter la lente désertion des Églises instituées ? Tant d'autres ont été poussés en vain, que l'on pourrait en douter. Voici cependant un ouvrage qui, prenant en toute rigueur cette question récurrente de la désaffection des hommes d'aujourd'hui par rapport aux Églises et, centralement, celles de la Réforme, tente de nouer une nouvelle alliance entre la religiosité des temps « postmodernes », et les composantes fondamentales de la foi chrétienne. Tâche complexe, qui suppose une remontée aux sources de ce qui apparaît de plus en plus comme un divorce sans appel. Mais quête d'autant plus urgente qu'elle atteste, dès la Réforme, d'un procès d'individuation, confiant à chaque croyant le privilège d'interprétation du Texte. Au risque d'entraîner un mouvement de libre inspiration, et d'enthousiasme, vite dénoncé par Luther et ses compagnons en rupture de ban. En l'âge des Modernes, la démarcation ira croissant entre « la vie quotidienne et le règles de l'Église ». Triomphe "de la liberté du sujet autonome", note Gilles Bourquin, qui en maximise les conséquences. Et sans doute le lecteur pourrait-il ne pas agréer en totalité aux figures du sujet ainsi mis en lumière. Individuation ne signifie pas ipso facto « retranchement de 
type individualiste », non plus qu'" absolutisation de l'homme». Et moins encore, ce "refus de la blessure, du procès, de l'imparfait, de l'incertain, du conflictuel», dont l'auteur « crédite » tel sujet en contexte de « sortie de religion ». Telles fêlures, en effet, semblent au principe de ces hommes de la modernité, désormais «sans qualité » dans un monde désenchanté. La "fonctionnalisation moderne de la religion» induit cette conscience aiguë des failles, dont la postmodernité accentue l'expression. Car le temps est désormais à la remise en cause du procès de rationalisation du monde, qui fait du sujet le lieu même de la plus grande "fragilité ». C'est cette fragilité qui va régler les rapports de l'homme avec le monde, soi-même, le temps de l'histoire et la passion d'autrui. La "dérégulation et l'atomisation de la religion " participent à l'évidence de cette formidable expansion du thème de l'intériorité, et qui n'est pas, ironie, sans rappeler la "découverte» de ce qui se nomma "chrétien intérieur» au XvII e siècle, dans un semblable moment historique de déréliction des institutions de religion. Mais alors que cette prise en charge de la subjectivité décidait de l'événement mystique, en la postmodernité, l'auteur identifie l'explosion d'un syncrétisme humaniste, agnostique, qui fonde une religiosité où la spontanéité est garante de l'authenticité du vécu « religieux ». La « valorisation de l'intensif subjectif » s'effectue « au détriment de l'extensif objectif ». Entendons par là que la spiritualité postmoderne, centrée sur les aspirations du moi, se confronte aux formes instituées et ritualisées des Églises, et à leurs prédications. Une spiritualité qui est " concept-limite ou résiduel de la religion », marquée de "fluidification et d'irrationalisme», et relevant d'une "approche expériencielle et pragmatique " du moi. Même si l'on peut s'interroger sur ce que recouvre cette référence au pragmatisme (stratégie de survie, adaptation fonctionnelle et gratifiante, ajustement, etc.), il est clair que telle spiritualité est ici fondée en l'expérience d'une existence singulière, et que cette existence vaut référence ultime. Le problème est alors, pour l'auteur, de penser «l'articulation de la religion objective, garante de la transmission de l'héritage, à sa réception subjective ». Il y va, en effet, du devenir même de l'Église, et de son témoignage aujourd'hui inaudible, quand le « religieux » risque d'apparaître comme phénomène culturel parmi d'autres.

2 Cette religiosité contemporaine s'ancre dans un substrat anthropologique qu'il convient donc d'analyser en profondeur, ses tenants et aboutissants, pour tester la possibilité d'une conjonction de l'existence singulière et de la forme ecclésiale collective. Forme ne signifie pas seulement institution, mais s'entend comme site de tous les « biens de salut » offerts aux croyants. La réflexion anthropologique se situe ici dans le sillage de Georg Simmel, qui étudia au début du xxe siècle le fondement de la spiritualité au centre vif de l'existence de l'homme. Et ce n'est pas le moindre des paradoxes de l'ouvrage, mais combien fécond, de solliciter le regard pénétrant de l'auteur de La religion (1912, trad. fr. Circé, 1998) et de Rembrandt (1916, trad. fr., 1994), pour définir la configuration postmoderne de la religiosité. Le lecteur s'enchante d'une aussi précieuse mise en perspective simmelienne de la genèse de la spiritualité, engagée dans une conception vitaliste fermement étayée. Pour le philosophe allemand, « ce sont les catégories du sujet qui déterminent ses rapports au monde, et non l'inverse». Catégories: dispositions affectives, psychosociales, cognitives, qui instituent l'homme en son unicité. Simmel abonde ainsi sans limite le pôle " phénoménal » par rapport au pôle «transcendantal » et, par cette " exaltation intellectuelle de la vitalité », livre une conception évolutionniste de la vie, qui signifie son "autoréalisation effrénée ». Dès lors, la vie en elle-même est une dynamique qui ne cesse de se dépasser, et chaque être est voué à transgresser ses propres limites. Chaque être porte en lui son principe de 
transcendance. Si, pour Simmel, «chaque moment de la vie est la vie entière », alors la vie s'identifie bien à l'« absolu ", car "il n'est rien qui lui soit supérieur, si ce n'est le fait de se surmonter sans cesse elle-même ». Simmel récuse ainsi toute téléologie qui ne serait pas strictement immanente : «La vie n'est précédée ni orientée par rien d'autre qu'elle-même ». Aussi bien, il n'est de spiritualité que comme " émanation » ou reprise de la vie. "Efflorescence spontanée ", articulée au plus profond de la singularité de chaque être, qui vaut attestation universelle. Par là, on conçoit que Simmel, rejetant toute pensée des " essences ", privilégie les seules "variations infiniment progressives de l'étant ». Rien de ce qui ressortit à l'ordre du religieux n'est pensable en d'autres termes que strictement, et seulement, humains. La spiritualité est l'indice ultime, et l'injonction, de la vitalité à raison d'homme. La transcendance ne peut alors se concevoir que comme dépassement par l'être humain de son propre être, et résorbé en sa sphère propre d'action.

3 La distance semble bien alors insurmontable entre la conception anthropologique simmelienne de la spiritualité, qui ouvre l'homme à lui-même, en ses limites et ses transgressions profanes, - et la théologie chrétienne, dont Bourquin rappelle que si la Parole est bien "travaillée à même le vécu», et, en tant que telle, «recherchée ou reçue », elle renvoie « ultimement à Dieu ». Elle est, pour reprendre la belle formule de l'auteur, "l'excès qui fait face à la conscience, confrontée à "plus-que-soi" ». Mais la philosophie vitaliste ne méconnaît pas, tant s'en faut, ce face-à-face de soi à l'autre : le principe d'altérité fonde la personnalité comme «catégorie subjective englobant des rapports au monde", et donc à tout autre. Au tout-autre. C'est en ce sens que la spiritualité est «la prise en charge intégrée des conditions existentielles de la vie humaine ", en sa vulnérabilité, sa quête de sens, ses stratégies de survie, dans l'indépendance absolue «de toute adhésion à un référentiel religieux». Et l'on comprend pourquoi Simmel est notre (post)contemporain, dès lors qu'il signifie, dans le concept de vie, quelque "désordre partiel mais volontaire", reflet, note le théologien protestant, "d'une image instantanée de l'état inachevé de la vie », où tous les possibles adviennent, et les "sentiments contrastés qui fondent la "personnalité" ". $\mathrm{Au}$ « caractère sui generis de l'être humain » correspond la religiosité comme «forme pure, non déductible d'aucune autre », qui lui serait extérieure.

La théorie vitaliste de la religion "insère la catégorie religieuse dans l'entier déploiement de la vie humaine dont elle procède à part entière ", résume l'auteur. Pour lui, il convient de prendre au sérieux ce défi d'interprétation qui pose la religion comme l'existence élevée « au degré supérieur où elle serait pour ainsi dire orientée vers l'intérieur ». Et s'opposerait ainsi à la tradition/transmission religieuses dans le contexte d'une Église déchue. Lorsque Simmel soutient la thèse de la religion à partir du seul argument existentiel et anthropologique, et la déleste de toute transcendance, le théologien tente de définir un concept de spiritualité qui puisse "réinscrire l'opérativité des affirmations théologiques au cœur de la vie, sans pour autant les dissoudre dans la vie». Tel est le propos central de l'ouvrage. Sans doute est-ce la condition pour qu'un véritable « réveil » religieux se déploie, sans que s'ensuive une explosion d'enthousiasmes et d'illuminations non maîtrisées par l'institution. Si la spiritualité relève bien « de l'humain comme tel (...) se surpassant et se réappropriant sans cesse lui-même", la révélation, en christianisme, "n'est pas réductible à un immanentisme vitaliste ». L'excès, cette formule pour dire le surgissement de la Parole 
décentrée radicalement de la vie en son « humanité », ne peut être ramené «à l'espace anthropologique ».

5 En d'autres termes, à ce que Simmel conçoit comme "habitation", moment de la spiritualité parvenue à la plénitude de son intensité, Bourquin propose en contrepoint l'impératif de la Révélation, et de sa source divine. La vie, dans la conception simmelienne, "n'est jamais exacte et sans reste ", et tout " objet qu'elle produit doit nécessairement lui faire face, étant à la fois le résultat et l'aiguillon de (son) autotranscendance ». Et Simmel pousse l'argument vitaliste jusqu'à sa plus forte tension : «L'assurance absolue de l'être religieux ne peut être garantie que si celle-ci se détache de tout fondement transcendant pour ne s'appuyer que sur lui-même »- nulle "localisation" extérieure ne vaut. L'auteur ne peut, à coup sûr, convenir de cet argument. Il lui oppose le nécessaire "décentrement» de la spiritualité de sa source "vitale » afin de la rapporter à la " source religieuse extrinsèque ». La vérité ne relève pas de la raison humaine, mais « renvoie à l'ultra-topique de l'Esprit, à Dieu lui-même dans son rapport à l'humain », quand Simmel laissait « en suspens » l'existence de Dieu. Positions inconciliables, au-delà de la volonté, pour le théologien, de retrouver le temps perdu de la vie?

6 Aussi bien l'auteur part-il du concept d'autotranscendance développé par Simmel, pour en reverser l'économie symbolique au compte d'une Révélation reçue par le croyant en situation de "religiosité » effervescente. Car celle-ci, chez le philosophe allemand, se dit «divinisation » de la vie, portée à son absolu. C'est en ce point précis qu'immanence anthropologique et transcendance divine peuvent se répondre, et que peuvent se rejoindre la dimension ascendante de la spiritualité simmelienne, et la dimension descendante de la théologie de la Révélation. Il ne faut pas combler le hiatus qui sépare Simmel de la Révélation, mais, pour l'auteur, « habiter » en connaissance de cause cette impossible relation, et cependant la seule à faire, si l'on peut dire, foi. Selon lui, il ne fait pas de doute que, si «la reconduction du divin à l'anthropologique ne peut que mener à l'anéantissement du divin », en sens inverse, « la reconduction trop directe de l'anthropologique au divin supprime la relativité de l'homme et surévalue la théologie en discours inspiré et infaillible ». Entre la radicalité « mystique » de la spiritualité chez Simmel, et la Révélation dans la théologie chrétienne, majorée en son versant protestant, demeure sans doute un espace béant, dont Nietzsche, en son Antéchrist donne la raison, ainsi résumée par l'auteur : «L'Évangile authentique n'est autre que la spontanéité religieuse vécue dans la négation de toute doctrine et de tout rite », et la doctrine paulinienne est alors «l'antithèse même de l'Évangile ». Jamais sans doute l'écart ne fut plus attesté entre l'injonction anthropologique et l'impératif théologique. Bourquin ne méconnaît pas «l'ancrage inéluctable de la théologie » dans le réel du monde et des sujets qui le fondent et refondent en permanence, mais il maintient « la spécificité propre de l'élément théologique ». S'il ne conteste pas que la Parole, telle qu'annoncée dans les églises de la Réforme, «n'articule plus suffisamment la révélation objective à la révélation subjective ", et s'il convient que la révélation «prend corps à même l'expérience croyante ", il oppose, à cette véritable incarnation de la spiritualité dans le vécu des sujets, la grâce " faisant irruption dans le monde, à partir d'un projet excédant le monde ", ainsi régénéré par ce "surpassement » même. Si l'on voit bien la différence fondamentale entre la position médiane du théologien protestant, et la conception kirekegaardienne du divin - qui n'a de rapport à l'histoire "que dans l'irruption d'une différence absolue", qui fait scandale et paradoxe -, le lecteur retiendra cette volonté rigoureuse de penser la «pénétration de l'intentionnalité 
divine dans l'épaisseur du réel », contre une anthropologie qui articulerait la fragilité des « intentions concrètes ", et leur " ambivalence ", à la blessure "d'un mal radical ». Mais si le vécu simmelien est capable de penser le mal en telle radicalité, c'est de rapatrier sans cesse en sa propre économie tout le tragique d'une existence sans autre garant qu'elle-même, et autre fondement que son propre déploiement. Le vitalisme de Simmel ne semble pas pouvoir, malgré le raffinement de l'analyse proposée par Bourquin, laisser place à une intervention de surplomb, qui dirait Dieu, quand il ne faut entendre ici que l'homme en son existence paroxystique aussitôt assumée en sa qualité profane.

7 Un dialogue peut alors s'établir entre un tel vitalisme et la théologie de la Révélation, mais comme il en irait de deux énoncés parallèles, ne se rejoignant qu'en un improbable infini. Si pour le théologien la spiritualité est la « centration vitale de tout ce qui provient d'une source excentrée ", dont procède le libre arbitre, Simmel dit la liberté "non pas une invention de soi mais la reconnaissance d'un appel inscrit au cœur même de la personnalité qu'il s'agit d'accomplir». Quant au destin, "même envoyé par Dieu, (il) ne pourrait pas épouser si absolument la vie, s'il n'était pas la cause et l'effet de sa singularité » (Rembrandt, que je cite à partir de l'éd. Circé, p. 164). Bourquin sait prendre la mesure de l'extrême distance entre les deux conceptions de la spiritualité, vitaliste et théologique - et, dans la quête d'une articulation capable de régénérer la prédication Réformée, sait cependant, d'un savoir aussi clair, combien cette quête de défis en défis va s'épuisant. Mais c'est bien cela même qui donne à l'ouvrage son rythme et sa raison - sa passion. 